\title{
Crohn's disease is caused by a failure of the innate immune response with major abnormalities in macrophage biology
}

\author{
A W Segal \\ From 5th European Workshop on Immune-Mediated Inflammatory Diseases \\ Sitges-Barcelona, Spain. 1-3 December 2010
}

Crohn's disease is characterized by chronic inflammation primarily affecting the gastrointestinal tract. We tested the hypothesis that the disease is a form of immunodeficiency with an underlying impairment of the early innate immune response.

We investigated inflammatory responses in patients and controls by quantifying neutrophil recruitment and cytokine production following acute trauma. Neutrophil recruitment was diminished in Crohn's patients following trauma to the large and small bowel, and skin. This was associated with lower production of interleukin- 8 and interleukin-1 $\beta$. To determine the mechanism of this abnormality, we examined cytokine secretion by cultured monocyte-derived macrophages after exposure to various inflammatory mediators, and to E.coli, and found it to be reduced in Crohn's patients.

We then assessed local inflammatory, vascular changes, neutrophil accumulation in, and bacterial clearance from, the skin in response to subcutaneous injection of heatkilled E. coli. All were impaired in Crohn's disease. None of these abnormalities was related to polymorphisms in NOD2.

mRNA profiles were then determined in macrophages from Crohn's patients before and after exposure to E. coli. Markedly abnormal increased and decreased expression of a number of important genes has been observed in clusters of Crohn's patients.

We have also identified two enormous families with Crohn's disease, one with 50, and the other with 22, affected individuals. The results of linkage, expression and exome sequencing studies will be discussed.

Centre for Molecular Medicine, University College London, London, UK
These studies demonstrated that Crohn's patients possess a constitutionally weak innate immune response. We propose that leads to impaired removal of intestinal contents that breach the mucosal barrier of the bowel wall, resulting in granuloma formation and chronic inflammation. Current treatment of secondary chronic inflammation might only be symptomatic, exaggerating the underlying lesions and promoting disease chronicity.

Published: 25 November 2010

\section{References}

1. Marks DJ, et al: Defective acute inflammation in Crohn's disease: a clinical investigation. Lancet 2006, 367(9511):668-78.

2. Smith AM, et al: Disordered macrophage cytokine secretion underlies impaired acute inflammation and bacterial clearance in Crohn's disease. J Exp Med. 2009, 206(9):1883-97.

doi:10.1186/1479-5876-8-S1-16

Cite this article as: Segal: Crohn's disease is caused by a failure of the innate immune response with major abnormalities in macrophage biology. Journal of Translational Medicine 2010 8(Suppl 1):16. 